\title{
On split common solution problems: new nonlinear feasible algorithms, strong convergence results and their applications
}

Zhenhua $\mathrm{He}^{1}$ and Wei-Shih Du${ }^{2^{*}}$

\section{"Correspondence:}

wsdu@nknucc.nknu.edu.tw

2Department of Mathematics,

National Kaohsiung Normal

University, Kaohsiung, 824, Taiwan

Full list of author information is

available at the end of the article

\begin{abstract}
In this paper, we study and give examples for classes of generalized contractive mappings. We establish some new strong convergence theorems of feasible iterative algorithms for the split common solution problem (SCSP) and give some applications of these new results.

MSC: $47 \mathrm{H} 06 ; 47 \mathrm{~J} 25 ; 47 \mathrm{H} 09 ; 65 \mathrm{~K} 10$

Keywords: Lipschitzian; demicontractive mapping; pseudocontractive mapping; quasi-nonexpansive mapping; nonexpansive mapping; split common solution problem (SCSP); iterative algorithm; strong convergence theorem
\end{abstract}

\section{Introduction and preliminaries}

Let $K$ be a closed convex subset of a real Hilbert space $H$ with the inner product $\langle\cdot, \cdot\rangle$ and the norm $\|\cdot\|$. The following inequalities are known and useful.

- $\|x+y\|^{2} \leq\|y\|^{2}+2\langle x, x+y\rangle$

- $\|x-y\|^{2}=\|x\|^{2}+\|y\|^{2}-2\langle x, y\rangle$ for all $x, y \in H$;

- $\|\alpha x+(1-\alpha) y\|^{2}=\alpha\|x\|^{2}+(1-\alpha)\|y\|^{2}-\alpha(1-\alpha)\|x-y\|^{2}$ for all $x, y \in H$ and $\alpha \in[0,1]$.

For each point $x \in H$, there exists a unique nearest point in $K$, denoted by $P_{K} x$, such that

$$
\left\|x-P_{K} x\right\| \leq\|x-y\| \quad \text { for all } y \in K .
$$

The mapping $P_{K}$ is called the metric projection from $H$ onto $K$. It is well known that $P_{K}$ has the following properties:

(i) $\left\langle x-y, P_{K} x-P_{K} y\right\rangle \geq\left\|P_{K} x-P_{K} y\right\|^{2}$ for every $x, y \in H$.

(ii) For $x \in H$ and $z \in K, z=P_{K} x \Leftrightarrow\langle x-z, z-y\rangle \geq 0$ for all $y \in K$.

(iii) For $x \in H$ and $y \in K$,

$$
\left\|y-P_{K} x\right\|^{2}+\left\|x-P_{K} x\right\|^{2} \leq\|x-y\|^{2} .
$$

Let $H_{1}$ and $H_{2}$ be two Hilbert spaces. Let $A: H_{1} \rightarrow H_{2}$ and $A^{*}: H_{2} \rightarrow H_{1}$ be two bounded linear operators. $A^{*}$ is called the adjoint operator (or adjoint) of $A$ if

$$
\langle A z, w\rangle=\left\langle z, A^{*} w\right\rangle \quad \text { for all } z \in H_{1} \text { and } w \in H_{2} \text {. }
$$

\section{Springer}

○2014 He and Du; licensee Springer. This is an Open Access article distributed under the terms of the Creative Commons Attribution License (http://creativecommons.org/licenses/by/2.0), which permits unrestricted use, distribution, and reproduction in any medium, provided the original work is properly cited. 
It is known that the adjoint operator of a bounded linear operator on a Hilbert space always exists and is bounded linear and unique. Moreover, it is not hard to show that if $A^{*}$ is an adjoint operator of $A$, then $\|A\|=\left\|A^{*}\right\|$. The symbols $\mathbb{N}$ and $\mathbb{R}$ are used to denote the sets of positive integers and real numbers, respectively.

Let $H_{1}$ and $H_{2}$ be two real Hilbert spaces. Let $C$ be a closed convex subset of $H_{1}$ and $K$ be a closed convex subset of $H_{2}$. Let $T: C \rightarrow C$ with $\mathcal{F}(T) \neq \emptyset$ and $S: K \rightarrow K$ with $\mathcal{F}(S) \neq \emptyset$ be two mappings. Let $A: H_{1} \rightarrow H_{2}$ be a bounded linear operator. The mathematical model of the split common solution problem (SCSP in short) is defined as follows:

(SCSP) Find $p \in C$ such that $T p=p$ and $u:=A p \in K$ satisfying $S u=u$.

In fact, SCSP contains several important problems as special cases and many authors have studied and introduced some new iterative algorithms for SCSP and presented some strong and weak convergence theorems for SCSP; see, for instance, [1-24] and the references therein. Motivated and inspired by their works, in this paper, we study and establish new strong convergence results by using new iterative algorithms of SCSP for pseudocontractive mappings and $k$-demicontractive mappings in Hilbert spaces.

The paper is divided into four sections. In Section 2, we study and give examples for classes of generalized contractive mappings. Some new strong convergence theorems of feasible iterative algorithms for SCSP are established in Section 3. Finally, some applications and further remarks for our new results are given in Section 4. Consequently, in this paper, some of our results are original and completely different from these known related results in the literature.

\section{Classes of generalized contractive mappings and their examples}

Let $T$ be a mapping with domain $\mathcal{D}(T)$ and range $\mathcal{R}(T)$ in a Hilbert space $H$. Recall that $T$ is said to be

(i) pseudocontractive if

$$
\langle T x-T y, x-y\rangle \leq\|x-y\|^{2}, \quad \forall x, y \in \mathcal{D}(T),
$$

or, equivalently,

$$
\|T x-T y\|^{2} \leq\|x-y\|^{2}+\|(I-T) x-(I-T) y\|^{2}, \quad \forall x, y \in \mathcal{D}(T) ;
$$

(ii) demicontractive if, for all $x \in \mathcal{D}(T)$ and $p \in \mathcal{F}(T)$,

$$
\langle T x-p, x-p\rangle \leq\|x-p\|^{2}
$$

or, equivalently,

$$
\|T x-p\|^{2} \leq\|x-p\|^{2}+\|(I-T) x\|^{2}
$$

(iii) $k$-demicontractive if there exists a constant $k \in[0,1)$ such that

$$
\|T x-p\|^{2} \leq\|x-p\|^{2}+k\|(I-T) x\|^{2} \text { for all } x \in \mathcal{D}(T) \text { and } p \in \mathcal{F}(T) ;
$$


(iv) quasi-nonexpansive if it is 0 -demicontractive, that is,

$$
\|T x-p\| \leq\|x-p\| \quad \text { for all } x \in \mathcal{D}(T) \text { and } p \in \mathcal{F}(T) ;
$$

(v) Lipschitzian if there exists $L>0$ such that

$$
\|T x-T y\| \leq L\|x-y\|, \quad \forall x, y \in \mathcal{D}(T)
$$

(vi) nonexpansive if it is Lipschitzian with $L=1$;

(vii) contractive if it is Lipschitzian with $L<1$.

A Banach space $(X,\|\cdot\|)$ is said to satisfy Opial's condition if, for each sequence $\left\{x_{n}\right\}$ in $X$ which converges weakly to a point $x \in X$, we have

$$
\liminf _{n \rightarrow \infty}\left\|x_{n}-x\right\|<\liminf _{n \rightarrow \infty}\left\|x_{n}-y\right\|, \quad \forall y \in X, y \neq x
$$

It is well known that any Hilbert space satisfies Opial's condition.

Definition 2.1 (see [2]) Let $K$ be a nonempty closed convex subset of a real Hilbert space $H$ and $T$ be a mapping from $K$ into $K$. The mapping $T$ is said to be demiclosed if, for any sequence $\left\{x_{n}\right\}$ which weakly converges to $y$, and if the sequence $\left\{T x_{n}\right\}$ strongly converges to $z$, then $T y=z$.

Remark 2.1 In Definition 2.1, the particular case of demiclosedness at zero is frequently used in some iterative convergence algorithms, which is the particular case when $z=\theta$, the zero vector of $H$; for more details, one can refer to [2].

The following concept of zero-demiclosedness was introduced as follows.

Definition 2.2 (see [25, Definition 2.3]) Let $K$ be a nonempty closed convex subset of a real Hilbert space and $T$ be a mapping from $K$ into $K$. The mapping $T$ is called zerodemiclosed if $\left\{x_{n}\right\}$ in $K$ satisfying $\left\|x_{n}-T x_{n}\right\| \rightarrow 0$ and $x_{n} \rightarrow z \in K$ implies $T z=z$.

The following result was essentially proved in [25], but we give the proof for the sake of completeness and the reader's convenience.

Theorem 2.1 (see [25, Proposition 2.4]) Let $K$ be a nonempty closed convex subset of a real Hilbert space with zero vector $\theta$. Then the following statements hold.

(a) Let $T$ be a mapping from $K$ into $K$. Then $T$ is zero-demiclosed if and only if $I-T$ is demiclosed at $\theta$.

(b) Let $T$ be a nonexpansive mapping from $H$ into itself. If there is a bounded sequence $\left\{x_{n}\right\} \subset H$ such that $\left\|x_{n}-T x_{n}\right\| \rightarrow 0$ as $n \rightarrow 0$, then $T$ is zero-demiclosed.

Proof Obviously, the conclusion (a) holds. To see (b), since $\left\{x_{n}\right\}$ is bounded, there is a subsequence $\left\{x_{n_{k}}\right\} \subset\left\{x_{n}\right\}$ and $z \in H$ such that $x_{n_{k}} \rightarrow z$. One can claim $T z=z$. Indeed, if $T z \neq z$, it follows from Opial's condition that

$$
\begin{aligned}
\liminf _{k \rightarrow \infty}\left\|x_{n_{k}}-z\right\| & <\liminf _{k \rightarrow \infty}\left\|x_{n_{k}}-T z\right\| \\
& \leq \liminf _{k \rightarrow \infty}\left\{\left\|x_{n_{k}}-T x_{n_{k}}\right\|+\left\|T x_{n_{k}}-T z\right\|\right\}
\end{aligned}
$$




$$
\begin{aligned}
& =\liminf _{k \rightarrow \infty}\left\|T x_{n_{k}}-T z\right\| \\
& \leq \liminf _{k \rightarrow \infty}\left\|x_{n_{k}}-z\right\|,
\end{aligned}
$$

which is a contradiction. So $T z=z$ and hence $T$ is zero-demiclosed.

Now, we give some examples to show the existence of these generalized contractive mappings (i)-(vi) which also expound the relation between them.

Example A Let $H=\mathbb{R}$ with the absolute-value norm $|\cdot|$ and $C=[-2,0]$. Let $T: C \rightarrow C$ be defined by

$$
T x= \begin{cases}x^{2}-2 & \text { if } x \in[-1,0] \\ -1 & \text { if } x \in[-2,-1]\end{cases}
$$

Then $\mathcal{F}(T)=\{-1\}$. Since

$$
|T x-(-1)|^{2} \leq|x-(-1)|^{2}+\frac{1}{2}|T x-x|^{2} \quad \text { for all } x \in C,
$$

we know that $T$ is a $\frac{1}{2}$-demicontractive mapping. However, due to

$$
\left|T\left(-\frac{1}{2}\right)-(-1)\right|>\left|-\frac{1}{2}-(-1)\right|
$$

$T$ is not quasi-nonexpansive.

Example B Let $H=\mathbb{R}$ with the absolute-value norm $|\cdot|$ and $C=\left[\frac{1}{2}, 2\right]$. Let $T: C \rightarrow C$ be defined by

$$
T x=\frac{1}{x}, \quad \forall x \in C
$$

Then $\mathcal{F}(T)=\{1\}$. Since

$$
|T x-1|^{2} \leq|x-1|^{2}+\frac{3}{4}|T x-x|^{2} \quad \text { for all } x \in C
$$

$T$ is a $\frac{3}{4}$-demicontractive mapping. Moreover, $T$ is also a pseudocontractive mapping.

Example $\mathbf{C}$ Let $H=\mathbb{R}$ with the absolute-value norm $|\cdot|$. Let $T: H \rightarrow H$ be defined by

$$
T x= \begin{cases}-\sqrt{-(1+x)} & \text { if } x \leq-2 \\ x+1 & \text { if } x \geq-2\end{cases}
$$

It is easy to see that

$$
|T x-T y| \leq|x-y| \quad \text { for all } x, y \in H
$$

So $T$ is continuous nonexpansive with $\mathcal{F}(T)=\emptyset$. 
The following example shows that there exists a continuous quasi-nonexpansive mapping which is not nonexpansive.

Example D (see [8]) Let $H=\mathbb{R}$ with the absolute-value norm $|\cdot|$ and $C=[0,+\infty)$. Define $T: C \rightarrow C$ by

$$
T x=\frac{x^{2}+2}{1+x}, \quad \forall x \in C
$$

Obviously, $\mathcal{F}(T)=\{2\}$. It is easy to see that

$$
|T x-2|=\frac{x}{1+x}|x-2| \leq|x-2| \quad \text { for all } x \in C
$$

and

$$
\left|T(0)-T\left(\frac{1}{3}\right)\right|=\frac{5}{12}>\left|0-\frac{1}{3}\right| .
$$

Hence $T$ is a continuous quasi-nonexpansive mapping but not nonexpansive.

The following example shows that there exists a demicontractive mapping which is neither pseudocontractive nor $k$-demicontractive for all $k \in[0,1)$.

Example E Let $H=\mathbb{R}$ with the absolute-value norm $|\cdot|$. Let $T: H \rightarrow H$ be defined by

$$
T x= \begin{cases}x^{2}-x+1 & \text { if } x \in(-\infty, 1], \\ \frac{x^{2}+1}{1+x} & \text { if } x \in[1,+\infty) .\end{cases}
$$

Then $\mathcal{F}(T)=\{1\}$. Since

$$
|T x-1|^{2} \leq|x-1|^{2}+|T x-x|^{2} \quad \text { for all } x \in H,
$$

$T$ is a demicontractive mapping. However, $T$ is not a pseudocontractive mapping due to the fact that when $x=-3$ and $y=-2.5$, we have

$$
|T x-T y|^{2}>|x-y|^{2}+|(x-T x)-(y-T y)|^{2} .
$$

It is easy to see that $T$ is not a $k$-demicontractive mapping for all $k \in[0,1)$.

The following example shows that there exists a discontinuous pseudocontractive mapping which is not a demicontractive mapping.

Example F Let $H=\mathbb{R}$ with the absolute-value norm $|\cdot|$. Let $T: H \rightarrow H$ be defined by

$$
T x= \begin{cases}x^{2}+1 & \text { if } x \in(-\infty, 0], \\ -1-x^{2} & \text { if } x \in(0,+\infty) .\end{cases}
$$

Then $\mathcal{F}(T)=\emptyset$. Due to

$$
|T x-T y|^{2} \leq|x-y|^{2}+|(I-T) x-(I-T) y|^{2} \quad \text { for all } x \in H,
$$


we know that $T$ is a discontinuous pseudocontractive mapping but not a demicontractive mapping.

The following example shows that there exists a pseudocontractive mapping which is not $k$-demicontractive for all $k \in[0,1)$.

Example G Let $H=\mathbb{R}$ with the absolute-value norm $|\cdot|$. Let $T: H \rightarrow H$ be defined by

$$
T x= \begin{cases}2-x^{2} & \text { if } x \in[0,1] \\ 2-x & \text { if } x \in[1,2], \\ 0 & \text { if } x \in[1,+\infty) .\end{cases}
$$

Then $\mathcal{F}(T)=\{1\}$. Since

$$
|T x-T y|^{2} \leq|x-y|^{2}+|(I-T) x-(I-T) y|^{2} \quad \text { for all } x \in H,
$$

$T$ is a pseudocontractive mapping. It is easy to see that $T$ is not a $k$-demicontractive mapping for all $k \in[0,1)$.

The following example shows that there exists a discontinuous $k$-demicontractive mapping for some $k \in[0,1)$ as well as being demiclosed at $\theta$ which is neither pseudocontractive nor quasi-nonexpansive.

Example $\mathbf{H}$ Let $H=\mathbb{R}$ with the absolute-value norm $|\cdot|$ and $C=[-2,0]$. Let $T: C \rightarrow C$ be defined by

$$
T x= \begin{cases}x^{2}-2 & \text { if } x \in[-1,0], \\ -\frac{1}{8} & \text { if } x=-\frac{3}{2}, \\ -1 & \text { if } x \in\left[-2,-\frac{3}{2}\right) \cup\left(-\frac{3}{2},-1\right] .\end{cases}
$$

Then the following statements hold.

(a) $T$ is discontinuous $\frac{3}{4}$-demicontractive.

(b) $T$ is demiclosed at $\theta$.

(c) $T$ is not pseudocontractive.

(d) $T$ is not quasi-nonexpansive.

Proof Clearly, $\mathcal{F}(T)=\{-1\}$. Since

$$
|T x-(-1)|^{2} \leq|x-(-1)|^{2}+\frac{3}{4}|(I-T) x|^{2} \quad \text { for all } x \in C,
$$

$T$ is a discontinuous $\frac{3}{4}$-demicontractive mapping and (a) is proved. Now, we verify (b). In fact, let $\left\{x_{n}\right\} \subset[-2,0]$ with $x_{n} \rightarrow z$ and $x_{n}-T x_{n} \rightarrow 0$ as $n \rightarrow \infty$. If all $x_{n} \in[-1,0]$, we can prove $T z=z$ and $z=-1 \in F(T)$ easily. If there exists a subsequence $\left\{x_{n_{k}}\right\} \subset[-2,-1]$, then, from $x_{n}-T x_{n} \rightarrow 0$ as $n \rightarrow \infty$, we can find a subsequence $\left\{x_{n_{k_{i}}}\right\}$ of $\left\{x_{n_{k}}\right\}$ such that $x_{n_{k_{i}}} \neq-\frac{3}{2}$ for all $i$. Hence we have

$$
|z-(-1)| \leq\left|z-x_{n_{k_{i}}}\right|+\left|x_{n_{k_{i}}}-T x_{n_{k_{i}}}\right|+\left|T x_{n_{k_{i}}}-(-1)\right| \rightarrow 0 \quad \text { as } i \rightarrow \infty,
$$


which implies $z=-1 \in \mathcal{F}(T)$. To see (c) and (d), note that

$$
\left|T\left(-\frac{3}{2}\right)-T\left(-\frac{25}{16}\right)\right|^{2}>\left|-\frac{3}{2}-\left(-\frac{25}{16}\right)\right|^{2}+\left|(I-T)\left(-\frac{3}{2}\right)-(I-T)\left(-\frac{25}{16}\right)\right|^{2}
$$

and

$$
\left|T\left(-\frac{3}{2}\right)-(-1)\right|>\left|\left(-\frac{3}{2}\right)-(-1)\right|,
$$

so $T$ is neither pseudocontractive nor quasi-nonexpansive. The proof is completed.

\section{New feasible iterative algorithms for SCSP and strong convergence theorems}

In this section, we establish some new strong convergence theorems by using feasible iterative algorithms for SCSP.

Theorem 3.1 Let $H_{1}$ and $H_{2}$ be two real Hilbert spaces and $\theta_{i}$ be the zero vector of $H_{i}$ for $i=1,2$. Let $C$ be a nonempty closed convex subset of $H_{1}$ and $A: H_{1} \rightarrow H_{2}$ be a bounded linear operator with its adjoint $B$. Let $T: C \rightarrow C$ be a Lipschitzian pseudocontractive mapping with Lipschitz constant $L>0$ and $\mathcal{F}(T) \neq \emptyset$, and let $S: H_{2} \rightarrow H_{2}$ be a k-demicontractive mapping with $\mathcal{F}(S) \neq \emptyset$ which is demiclosed at $\theta_{2}$. Let $C_{1}=C$ and $\left\{x_{n}\right\}$ be a sequence generated by the following algorithm:

$$
\left\{\begin{array}{l}
x_{1} \in C_{1} \quad \text { chosen arbitrarily, } \\
y_{n}=(1-\alpha) x_{n}+\alpha T x_{n}, \\
z_{n}=\beta x_{n}+(1-\beta) T y_{n}, \\
w_{n}=P_{C}\left(z_{n}+\xi B(S-I) A z_{n}\right), \\
C_{n+1}=\left\{v \in C_{n}:\left\|w_{n}-v\right\| \leq\left\|z_{n}-v\right\| \leq\left\|x_{n}-v\right\|\right\}, \\
x_{n+1}=P_{C_{n+1}}\left(x_{1}\right), \quad \forall n \in \mathbb{N},
\end{array}\right.
$$

where $0<1-\beta<\alpha<\frac{1}{2 \sqrt{1+L^{2}}}, \xi \in\left(0, \frac{1-k}{\|B\|^{2}}\right)$ and $P_{C_{n}}$ is the projection operator from $H_{1}$ into $C_{n}$ for $n \in \mathbb{N}$. Suppose that

$$
\Omega=\{p \in \mathcal{F}(T): A p \in \mathcal{F}(S)\} \neq \emptyset .
$$

Then there exists $q \in \Omega$ such that

(a) $x_{n} \rightarrow q$ as $n \rightarrow \infty$,

(b) $A x_{n} \rightarrow$ Aq as $n \rightarrow \infty$.

Proof We will show the conclusion by proceeding with the following steps.

Step 1 . For any $p \in \Omega$, we prove

$$
\left\|w_{n}-p\right\|^{2} \leq\left\|z_{n}-p\right\|^{2}-\xi\left(1-k-\xi\|B\|^{2}\right)\left\|(S-I) A z_{n}\right\|^{2} .
$$

Indeed, since

$$
\begin{aligned}
& \left\|w_{n}-p\right\|^{2} \\
& \quad \leq\left\|z_{n}+\xi B(S-I) A z_{n}-p\right\|^{2}
\end{aligned}
$$




$$
\begin{aligned}
& =\left\|z_{n}-p\right\|^{2}+\left\|\xi B(S-I) A z_{n}\right\|^{2}+2 \xi\left\langle z_{n}-p, B(S-I) A z_{n}\right\rangle \\
& =\left\|z_{n}-p\right\|^{2}+\left\|\xi B(S-I) A z_{n}\right\|^{2}+2 \xi\left\langle A z_{n}-A p,(S-I) A z_{n}\right\rangle \\
& =\left\|z_{n}-p\right\|^{2}+\left\|\xi B(S-I) A z_{n}\right\|^{2}+2 \xi\left\langle A z_{n}-A p+(S-I) A z_{n}-(S-I) A z_{n},(S-I) A z_{n}\right\rangle \\
& =\left\|z_{n}-p\right\|^{2}+\left\|\xi B(S-I) A z_{n}\right\|^{2}+2 \xi\left\langle S A z_{n}-A p,(S-I) A z_{n}\right\rangle-2 \xi\left\|(S-I) A z_{n}\right\|^{2} \\
& \leq\left\|z_{n}-p\right\|^{2}+\xi^{2}\|B\|^{2}\left\|(S-I) A z_{n}\right\|^{2}+2 \xi\left\langle S A z_{n}-A p,(S-I) A z_{n}\right\rangle-2 \xi\left\|(S-I) A z_{n}\right\|^{2}
\end{aligned}
$$

and

$$
\begin{aligned}
& 2 \xi\left\langle S A z_{n}-A p,(S-I) A z_{n}\right\rangle \\
& \quad=\xi\left\{\left\|S A z_{n}-A p\right\|^{2}+\left\|(S-I) A z_{n}\right\|^{2}-\left\|A z_{n}-A p\right\|^{2}\right\} \\
& \quad \leq \xi\left\{\left\|A z_{n}-A p\right\|^{2}+k\left\|(S-I) A z_{n}\right\|^{2}+\left\|(S-I) A z_{n}\right\|^{2}-\left\|A z_{n}-A p\right\|^{2}\right\} \\
& \quad \leq \xi\left\{-\left\|A z_{n}-A z_{n}\right\|^{2}+k\left\|(S-I) A z_{n}\right\|^{2}+\left\|(S-I) A z_{n}\right\|^{2}\right\} \\
& \quad=\xi\left\{k\left\|(S-I) A z_{n}\right\|^{2}+\left\|(S-I) A z_{n}\right\|^{2}\right\},
\end{aligned}
$$

we get

$$
\left\|w_{n}-p\right\|^{2} \leq\left\|z_{n}-p\right\|^{2}-\xi\left(1-k-\xi\|B\|^{2}\right)\left\|(S-I) A z_{n}\right\|^{2},
$$

and our desired result is proved.

Step 2. We prove

$$
\left\|z_{n}-p\right\| \leq\left\|x_{n}-p\right\| \quad \text { for all } n \in \mathbb{N} \text {. }
$$

For any $n \in \mathbb{N}$, by (3.1), we have

$$
\begin{aligned}
\left\|z_{n}-p\right\|^{2} & \\
= & \beta\left\|x_{n}-p\right\|^{2}+(1-\beta)\left\|T y_{n}-p\right\|^{2}-(1-\beta) \beta\left\|T y_{n}-x_{n}\right\|^{2} \\
\leq & \beta\left\|x_{n}-p\right\|^{2}+(1-\beta)\left\|y_{n}-p\right\|^{2}+(1-\beta)\left\|T y_{n}-y_{n}\right\|^{2}-(1-\beta) \beta\left\|T y_{n}-x_{n}\right\|^{2} \\
\leq & \beta\left\|x_{n}-p\right\|^{2}+(1-\beta)\left((1-\alpha)\left\|x_{n}-p\right\|^{2}+\alpha\left\|T x_{n}-x_{n}\right\|^{2}-(1-\alpha) \alpha\left\|T x_{n}-x_{n}\right\|^{2}\right) \\
& +(1-\beta)\left\|T y_{n}-y_{n}\right\|^{2}-(1-\beta) \beta\left\|T y_{n}-x_{n}\right\|^{2} \\
\leq & \left\|x_{n}-p\right\|^{2}+(1-\beta)\left(\alpha\left\|T x_{n}-x_{n}\right\|^{2}-(1-\alpha) \alpha\left\|T x_{n}-x_{n}\right\|^{2}\right)-(1-\beta) \beta\left\|T y_{n}-x_{n}\right\|^{2} \\
& +(1-\beta)\left\|(1-\alpha)\left(x_{n}-T y_{n}\right)+\alpha\left(T x_{n}-T y_{n}\right)\right\|^{2} \\
\leq & \left\|x_{n}-p\right\|^{2}+(1-\beta)\left(\alpha\left\|T x_{n}-x_{n}\right\|^{2}-(1-\alpha) \alpha\left\|T x_{n}-x_{n}\right\|^{2}\right)-(1-\beta) \beta\left\|T y_{n}-x_{n}\right\|^{2} \\
& +(1-\beta)\left((1-\alpha)\left\|x_{n}-T y_{n}\right\|^{2}+\alpha\left\|T x_{n}-T y_{n}\right\|^{2}-(1-\alpha) \alpha\left\|T x_{n}-x_{n}\right\|^{2}\right) \\
\leq & \left\|x_{n}-p\right\|^{2}+(1-\beta)\left(\alpha\left\|T x_{n}-x_{n}\right\|^{2}-(1-\alpha) \alpha\left\|T x_{n}-x_{n}\right\|^{2}\right)-(1-\beta) \beta\left\|T y_{n}-x_{n}\right\|^{2} \\
& +(1-\beta)\left((1-\alpha)\left\|x_{n}-T y_{n}\right\|^{2}+\alpha L^{2}\left\|x_{n}-y_{n}\right\|^{2}-(1-\alpha) \alpha\left\|T x_{n}-x_{n}\right\|^{2}\right) \\
\leq & \left\|x_{n}-p\right\|^{2}+(1-\beta)\left(\alpha\left\|T x_{n}-x_{n}\right\|^{2}-(1-\alpha) \alpha\left\|T x_{n}-x_{n}\right\|^{2}\right)-(1-\beta) \beta\left\|T y_{n}-x_{n}\right\|^{2}
\end{aligned}
$$




$$
\begin{aligned}
& +(1-\beta)\left((1-\alpha)\left\|x_{n}-T y_{n}\right\|^{2}+\alpha^{3} L^{2}\left\|x_{n}-T x_{n}\right\|^{2}-(1-\alpha) \alpha\left\|T x_{n}-x_{n}\right\|^{2}\right) \\
= & \left\|x_{n}-p\right\|^{2}-(1-\beta)(\alpha+\beta-1)\left\|T y_{n}-x_{n}\right\|^{2} \\
& -(1-\beta) \alpha\left(1-2 \alpha-\alpha^{2} L^{2}\right)\left\|T x_{n}-x_{n}\right\|^{2} .
\end{aligned}
$$

Since $\alpha+\beta>1$ and $\alpha<\frac{1}{2 \sqrt{1+L^{2}}}$, from (3.4), we have $\left\|z_{n}-p\right\|^{2} \leq\left\|x_{n}-p\right\|^{2}$, or, equivalently,

$$
\left\|z_{n}-p\right\| \leq\left\|x_{n}-p\right\|
$$

Step 3. We show that $C_{n}$ is a nonempty closed convex set for any $n \in \mathbb{N}$.

For any $p \in \Omega$, by taking into account (3.2) and (3.5), we obtain

$$
\left\|w_{n}-p\right\| \leq\left\|z_{n}-p\right\| \leq\left\|x_{n}-p\right\| \quad \text { for all } n \in \mathbb{N} \text {. }
$$

So we know $\Omega \subset C_{n}$ and hence $C_{n} \neq \emptyset$ for all $n \in \mathbb{N}$. It is easy to verify that $C_{n}$ is closed and convex for all $n \in \mathbb{N}$.

Step 4. We prove that $\left\{x_{n}\right\}$ is a Cauchy sequence in $C$ and $x_{n} \rightarrow q$ as $n \rightarrow \infty$ for some $q \in C$.

Since $\Omega \subset C_{n+1} \subset C_{n}$ and $x_{n+1}=P_{C_{n+1}}\left(x_{1}\right) \subset C_{n}$, we get

$$
\left\|x_{n+1}-x_{1}\right\| \leq\left\|p-x_{1}\right\| \quad \text { for all } p \in \Omega
$$

and

$$
\left\|x_{n}-x_{1}\right\| \leq\left\|x_{n+1}-x_{1}\right\| \quad \text { for all } n \in \mathbb{N}
$$

which show that $\left\{x_{n}\right\}$ is bounded and $\left\{\left\|x_{n}-x_{1}\right\|\right\}$ is nondecreasing in $[0, \infty)$. So

$$
\lim _{n \rightarrow \infty}\left\|x_{n}-x_{1}\right\| \geq 0
$$

exists. For any $m, n \in \mathbb{N}$ with $m>n$, from $x_{m}=P_{C_{m}}\left(x_{1}\right) \subset C_{n}$ and (1.1), we have

$$
\left\|x_{m}-x_{n}\right\|^{2}+\left\|x_{1}-x_{n}\right\|^{2}=\left\|x_{m}-P_{C_{n}}\left(x_{1}\right)\right\|^{2}+\left\|x_{1}-P_{C_{n}}\left(x_{1}\right)\right\|^{2} \leq\left\|x_{m}-x_{1}\right\|^{2} .
$$

Inequality (3.6) implies

$$
\lim _{m, n \rightarrow \infty}\left\|x_{n}-x_{m}\right\|=0
$$

So $\left\{x_{n}\right\}$ is a Cauchy sequence. Clearly,

$$
\lim _{n \rightarrow \infty}\left\|x_{n+1}-x_{n}\right\|=0
$$

By the completeness of $C$, there exists $q \in C$ such that $x_{n} \rightarrow q$ as $n \rightarrow \infty$.

Step 5. Finally, we show that the following hold:

(i) $q \in \Omega$,

(ii) $A x_{n} \rightarrow A q$ as $n \rightarrow \infty$. 
For any $n \in \mathbb{N}$, since $x_{n+1}=P_{C_{n+1}}\left(x_{1}\right) \in C_{n+1} \subset C_{n}$, from (3.1), we have

$$
\left\|z_{n}-x_{n}\right\| \leq\left\|z_{n}-x_{n+1}\right\|+\left\|x_{n+1}-x_{n}\right\| \leq 2\left\|x_{n+1}-x_{n}\right\|
$$

and

$$
\left\|w_{n}-x_{n}\right\| \leq\left\|w_{n}-x_{n+1}\right\|+\left\|x_{n+1}-x_{n}\right\| \leq 2\left\|x_{n+1}-x_{n}\right\| .
$$

From inequalities (3.7), (3.8) and (3.9), we deduce

$$
\begin{aligned}
& \lim _{n \rightarrow \infty}\left\|z_{n}-x_{n}\right\|=0, \\
& \lim _{n \rightarrow \infty}\left\|w_{n}-x_{n}\right\|=0
\end{aligned}
$$

and hence

$$
\lim _{n \rightarrow \infty}\left\|w_{n}-z_{n}\right\|=0
$$

By taking into account (3.4) and (3.10), we get

$$
\begin{aligned}
& \alpha\left(1-2 \alpha-\alpha^{2} L^{2}\right)\left\|T x_{n}-x_{n}\right\|^{2}+(\alpha+\beta-1)\left\|T y_{n}-x_{n}\right\|^{2} \\
& \quad \leq \frac{1}{1-\beta}\left(\left\|x_{n}-p\right\|^{2}-\left\|z_{n}-p\right\|^{2}\right) \\
& \quad \leq \frac{2}{1-\beta}\left\|x_{n}-z_{n}\right\|\left\|x_{n}-p\right\| \rightarrow 0 \quad \text { as } n \rightarrow \infty
\end{aligned}
$$

So, we obtain

$$
\lim _{n \rightarrow \infty}\left\|T x_{n}-x_{n}\right\|=\lim _{n \rightarrow \infty}\left\|T y_{n}-x_{n}\right\|=0
$$

Since $x_{n} \rightarrow q$ as $n \rightarrow \infty$, from (3.12) and the continuity of the norm $\|\cdot\|$ and the Lipschitzian pseudocontractive mapping $T$, we can deduce that $T q=q$, namely $q \in \mathcal{F}(T)$. On the other hand, from (3.2) and (3.11), we have

$$
\begin{aligned}
& \xi\left(1-k-\xi\|B\|^{2}\right)\left\|(S-I) A z_{n}\right\|^{2} \\
& \quad \leq\left\|z_{n}-p\right\|^{2}-\left\|w_{n}-p\right\|^{2} \\
& \quad \leq\left\|z_{n}-w_{n}\right\|\left(\left\|z_{n}-p\right\|-\left\|w_{n}-p\right\|\right) \rightarrow 0 \quad \text { as } n \rightarrow \infty
\end{aligned}
$$

which yields that

$$
\lim _{n \rightarrow \infty}\left\|(S-I) A z_{n}\right\|=0
$$

Since the $k$-demicontractive mapping $S$ is demiclosed at $\theta_{2}$, taking into account $x_{n} \rightarrow q$, $A x_{n} \rightarrow A q,\left\|z_{n}-x_{n}\right\| \rightarrow 0$ and (3.13), we have

$$
A z_{n} \rightarrow A q
$$


and

$A q \in \mathcal{F}(S)$.

Hence we confirm $q \in \Omega$. The proof is completed.

By virtue of Theorem 3.1, we can establish the following:

(i) Strong convergence algorithms for the split common solution problem for Lipschitzian pseudocontractive mappings and nonexpansive mappings (see Corollary 3.1 below).

(ii) Strong convergence algorithms for the split common solution problem for Lipschitzian pseudocontractive mappings and quasi-nonexpansive mappings (see Corollary 3.2 below).

Corollary 3.1 Let $H_{1}$ and $H_{2}$ be two real Hilbert spaces and $\theta_{i}$ be the zero vector of $H_{i}$ for $i=1,2$. Let $C$ be a nonempty closed convex subset of $H_{1}$ and $A: H_{1} \rightarrow H_{2}$ be a bounded linear operator with its adjoint $B$. Let $T: C \rightarrow C$ be a Lipschitzian pseudocontractive mapping with Lipschitz constant $L>0$ and $\mathcal{F}(T) \neq \emptyset$, and let $S: H_{2} \rightarrow H_{2}$ be a nonexpansive mapping with $\mathcal{F}(S) \neq \emptyset$. Let $C_{1}=C$ and $\left\{x_{n}\right\}$ be a sequence generated by the following algorithm:

$$
\left\{\begin{array}{l}
x_{1} \in C_{1} \quad \text { chosen arbitrarily, } \\
y_{n}=(1-\alpha) x_{n}+\alpha T x_{n}, \\
z_{n}=\beta x_{n}+(1-\beta) T y_{n}, \\
w_{n}=P_{C}\left(z_{n}+\xi B(S-I) A z_{n}\right), \\
C_{n+1}=\left\{v \in C_{n}:\left\|w_{n}-v\right\| \leq\left\|z_{n}-v\right\| \leq\left\|x_{n}-v\right\|\right\}, \\
x_{n+1}=P_{C_{n+1}}\left(x_{1}\right), \quad \forall n \in \mathbb{N},
\end{array}\right.
$$

where $0<1-\beta<\alpha<\frac{1}{2 \sqrt{1+L^{2}}}, \xi \in\left(0, \frac{1}{\|B\|^{2}}\right)$ and $P_{C_{n}}$ is the projection operator from $H_{1}$ into $C_{n}$ for $n \in \mathbb{N}$. Suppose that

$$
\Omega=\{p \in \mathcal{F}(T): A p \in \mathcal{F}(S)\} \neq \emptyset \text {. }
$$

Then there exists $q \in \Omega$ such that

(a) $x_{n} \rightarrow q$ as $n \rightarrow \infty$,

(b) $A x_{n} \rightarrow$ Aq as $n \rightarrow \infty$.

Proof Since the mapping $S$ is nonexpansive, it is 0 -demicontractive. Hence the desired conclusion follows from Theorem 3.1 immediately by taking $k=0$.

Corollary 3.2 Let $H_{1}$ and $H_{2}$ be two real Hilbert spaces and $\theta_{i}$ be the zero vector of $H_{i}$ for $i=1$, 2. Let $C$ be a nonempty closed convex subset of $H_{1}$ and $A: H_{1} \rightarrow H_{2}$ be a bounded linear operator with its adjoint $B$. Let $T: C \rightarrow C$ be a Lipschitzian pseudocontractive mapping with Lipschitz constant $L>0$ and $\mathcal{F}(T) \neq \emptyset$, and let $S: H_{2} \rightarrow H_{2}$ be a quasi-nonexpansive mapping with $\mathcal{F}(S) \neq \emptyset$ which is demiclosed at $\theta_{2}$. Let $C_{1}=C$ and $\left\{x_{n}\right\}$ be a sequence gen- 
erated by the following algorithm:

$$
\left\{\begin{array}{l}
x_{1} \in C_{1} \quad \text { chosen arbitrarily, } \\
y_{n}=(1-\alpha) x_{n}+\alpha T x_{n}, \\
z_{n}=\beta x_{n}+(1-\beta) T y_{n}, \\
w_{n}=P_{C}\left(z_{n}+\xi B(S-I) A z_{n}\right), \\
C_{n+1}=\left\{v \in C_{n}:\left\|w_{n}-v\right\| \leq\left\|z_{n}-v\right\| \leq\left\|x_{n}-v\right\|\right\}, \\
x_{n+1}=P_{C_{n+1}}\left(x_{1}\right), \quad \forall n \in \mathbb{N},
\end{array}\right.
$$

where $0<1-\beta<\alpha<\frac{1}{2 \sqrt{1+L^{2}}}, \xi \in\left(0, \frac{1}{\|B\|^{2}}\right)$ and $P_{C_{n}}$ is the projection operator from $H_{1}$ into $C_{n}$ for $n \in \mathbb{N}$. Suppose that

$$
\Omega=\{p \in \mathcal{F}(T): A p \in \mathcal{F}(S)\} \neq \emptyset .
$$

Then there exists $q \in \Omega$ such that

(a) $x_{n} \rightarrow q$ as $n \rightarrow \infty$,

(b) $A x_{n} \rightarrow$ Aq as $n \rightarrow \infty$.

Example 3.1 Let $H_{1}=\mathbb{R}$ with the absolute-value norm $|\cdot|$. Let $H_{2}=\left[\frac{1}{\sqrt{2}}, \sqrt{2}\right]^{2}$ with the norm $\|\alpha\|=\left(a_{1}^{2}+a_{2}^{2}\right)^{\frac{1}{2}}$ for $\alpha=\left(a_{1}, a_{2}\right) \in H_{2}$ and the inner product $\langle\alpha, \beta\rangle=\sum_{i=1}^{2} a_{i} b_{i}$ for $\alpha=\left(a_{1}, a_{2}\right)$ and $\beta=\left(b_{1}, b_{2}\right) \in H_{2}$. Let $A: H_{1} \rightarrow H_{2}$ be defined by $A x=(x, x)$ for $x \in \mathbb{R}$. Then $A$ is a bounded linear operator with its adjoint operator $B z=z_{1}+z_{2}$ for $z=\left(z_{1}, z_{2}\right) \in H_{2}$. Clearly, $\|A\|=\|B\|=\sqrt{2}$. Let $C=\left[\frac{1}{\sqrt{2}}, \sqrt{2}\right]$. Let $T: C \rightarrow C$ and $S: H_{2} \rightarrow H_{2}$ be defined by

$$
T x=\frac{1}{x} \quad \text { for } x \in C
$$

and

$$
S z=\left(\frac{1}{z_{1}}, \frac{1}{z_{2}}\right) \quad \text { for } z=\left(z_{1}, z_{2}\right) \in H_{2},
$$

respectively. It is easy to see that

- $\mathcal{F}(T)=\{1\}$

- $\mathcal{F}(S)=\{(1,1)\}$

- $\Omega=\{p \in \mathcal{F}(T): A p \in \mathcal{F}(S)\}=\{1\} \neq \emptyset$;

- $T$ is a Lipschitzian pseudocontractive mapping with Lipschitz constant $L=\sqrt{2}$;

- $T$ and $S$ both are $\frac{3}{4}$-demicontractive mappings.

By using algorithm (3.1) with $0<1-\beta<\alpha<\frac{1}{2 \sqrt{3}}$ and $\xi \in\left(0, \frac{1}{8}\right)$, we can verify $x_{n} \rightarrow 1$ and $A x_{n} \rightarrow A(1)=(1,1) \in \mathcal{F}(S)$ as $n \rightarrow \infty$.

\section{Some applications and further remarks for Theorem 3.1}

Let $C$ be a nonempty subset of a Hilbert space $H$. Recall that a mapping $U: C \rightarrow C$ is said to be accretive if

$$
\langle U x-U y, x-y\rangle \geq 0 \quad \text { for all } x, y \in C .
$$


Obviously, $U: C \rightarrow C$ is accretive if and only if $I-U: C \rightarrow C$ is pseudocontractive. Moreover,

$$
\mathcal{F}(I-U)=U^{-1}(\theta):=\{x \in C: U x=\theta\},
$$

where $\theta$ is the zero vector of $H$.

At the end of this paper, by applying Theorem 3.1, we obtain the following:

(i) Strong convergence algorithms for the split common solution problem for Lipschitzian accretive mappings and demicontractive nonexpansive mappings (see Theorem 4.1 below).

(ii) Strong convergence algorithms for the split common solution problem for Lipschitzian accretive mappings and nonexpansive mappings (see Corollary 4.1 below).

(iii) Strong convergence algorithms for the split common solution problem for Lipschitzian accretive mappings and quasi-nonexpansive mappings (see Corollary 4.2 below).

Theorem 4.1 Let $H_{1}$ and $H_{2}$ be two real Hilbert spaces and $\theta_{i}$ be the zero vector of $H_{i}$ for $i=1$, 2. Let $A: H_{1} \rightarrow H_{2}$ be a bounded linear operator with its adjoint $B$ and $U: H_{1} \rightarrow H_{1}$ be a Lipschitzian accretive mapping with Lipschitz constant $L>0$ and $U^{-1}\left(\theta_{1}\right) \neq \emptyset$. Let $S: \mathrm{H}_{2} \rightarrow \mathrm{H}_{2}$ be a $k$-demicontractive mapping with $\mathcal{F}(S) \neq \emptyset$ which is demiclosed at $\theta_{2}$. Let $\left\{x_{n}\right\}$ be a sequence generated by the following algorithm:

$$
\left\{\begin{array}{l}
x_{1} \in H_{1} \quad \text { chosen arbitrarily, } \\
y_{n}=x_{n}-\alpha U x_{n}, \\
z_{n}=\beta x_{n}+(1-\beta)(I-U) y_{n}, \\
w_{n}=z_{n}+\xi B(S-I) A z_{n} \\
C_{n+1}=\left\{v \in C_{n}:\left\|w_{n}-v\right\| \leq\left\|z_{n}-v\right\| \leq\left\|x_{n}-v\right\|\right\} \\
x_{n+1}=P_{C_{n+1}}\left(x_{1}\right), \quad \forall n \in \mathbb{N}
\end{array}\right.
$$

where $0<1-\beta<\alpha<\frac{1}{2 \sqrt{1+L^{2}}}, \xi \in\left(0, \frac{1-k}{\|B\|^{2}}\right)$ and $P_{C_{n}}$ is the projection operator from $H_{1}$ into $C_{n}$ for $n \in \mathbb{N}$. Suppose that

$$
\Omega=\left\{p \in U^{-1}\left(\theta_{1}\right): A p \in \mathcal{F}(S)\right\} \neq \emptyset .
$$

Then there exists $q \in \Omega$ such that

(a) $x_{n} \rightarrow q$ as $n \rightarrow \infty$,

(b) $A x_{n} \rightarrow$ Aq as $n \rightarrow \infty$.

Proof Let $C_{1}=H_{1}$. Then the iterative process (4.1) can be rewritten as follows:

$$
\left\{\begin{array}{l}
x_{1} \in C_{1} \quad \text { chosen arbitrarily, } \\
y_{n}=(1-\alpha) x_{n}+\alpha(I-U) x_{n}, \\
z_{n}=\beta x_{n}+(1-\beta)(I-U) y_{n}, \\
w_{n}=z_{n}+\xi B(S-I) A z_{n}, \\
C_{n+1}=\left\{v \in C_{n}:\left\|w_{n}-v\right\| \leq\left\|z_{n}-v\right\| \leq\left\|x_{n}-v\right\|\right\}, \\
x_{n+1}=P_{C_{n+1}}\left(x_{1}\right), \quad \forall n \in \mathbb{N} .
\end{array}\right.
$$


Set $T:=I-U$, then $\mathcal{F}(T)=U^{-1}\left(\theta_{1}\right)$ and $T$ is a Lipschitzian pseudocontractive mapping with Lipschitz constant $1+L$. Therefore the desired conclusion follows from Theorem 3.1 immediately.

The following interesting results are immediate from Theorem 4.1.

Corollary 4.1 Let $H_{1}$ and $H_{2}$ be two real Hilbert spaces and $\theta_{i}$ be the zero vector of $H_{i}$ for $i=1,2$. Let $A: H_{1} \rightarrow H_{2}$ be a bounded linear operator with its adjoint $B$ and $U: H_{1} \rightarrow H_{1}$ be a Lipschitzian accretive mapping with Lipschitz constant $L>0$ and $U^{-1}\left(\theta_{1}\right) \neq \emptyset$. Let $S: H_{2} \rightarrow H_{2}$ be a quasi-nonexpansive mapping with $\mathcal{F}(S) \neq \emptyset$ which is demiclosed at $\theta_{2}$. Let $\left\{x_{n}\right\}$ be a sequence generated by the following algorithm:

$$
\left\{\begin{array}{l}
x_{1} \in H_{1} \quad \text { chosen arbitrarily, } \\
y_{n}=x_{n}-\alpha U x_{n}, \\
z_{n}=\beta x_{n}+(1-\beta)(I-U) y_{n}, \\
w_{n}=z_{n}+\xi B(S-I) A z_{n}, \\
C_{n+1}=\left\{v \in C_{n}:\left\|w_{n}-v\right\| \leq\left\|z_{n}-v\right\| \leq\left\|x_{n}-v\right\|\right\} \\
x_{n+1}=P_{C_{n+1}}\left(x_{1}\right), \quad \forall n \in \mathbb{N}
\end{array}\right.
$$

where $0<1-\beta<\alpha<\frac{1}{2 \sqrt{1+L^{2}}}, \xi \in\left(0, \frac{1}{\|B\|^{2}}\right)$ and $P_{C_{n}}$ is the projection operator from $H_{1}$ into $C_{n}$ for $n \in \mathbb{N}$. Suppose that

$$
\Omega=\left\{p \in U^{-1}\left(\theta_{1}\right): A p \in \mathcal{F}(S)\right\} \neq \emptyset \text {. }
$$

Then there exists $q \in \Omega$ such that

(a) $x_{n} \rightarrow q$ as $n \rightarrow \infty$,

(b) $A x_{n} \rightarrow$ Aq as $n \rightarrow \infty$.

Corollary 4.2 Let $H_{1}$ and $H_{2}$ be two real Hilbert spaces and $\theta_{i}$ be the zero vector of $H_{i}$ for $i=1$, 2. Let $A: H_{1} \rightarrow H_{2}$ be a bounded linear operator with its adjoint B. Let $U: H_{1} \rightarrow H_{1}$ be a Lipschitzian accretive mapping with Lipschitz constant $L>0$ and $U^{-1}\left(\theta_{1}\right) \neq \emptyset$. Let $S: H_{2} \rightarrow H_{2}$ be a nonexpansive mapping with $\mathcal{F}(S) \neq \emptyset$. Let $\left\{x_{n}\right\}$ be a sequence generated by the following algorithm:

$$
\left\{\begin{array}{l}
x_{1} \in H_{1} \quad \text { chosen arbitrarily, } \\
y_{n}=x_{n}-\alpha U x_{n} \\
z_{n}=\beta x_{n}+(1-\beta)(I-U) y_{n}, \\
w_{n}=z_{n}+\xi B(S-I) A z_{n}, \\
C_{n+1}=\left\{v \in C_{n}:\left\|w_{n}-v\right\| \leq\left\|z_{n}-v\right\| \leq\left\|x_{n}-v\right\|\right\} \\
x_{n+1}=P_{C_{n+1}}\left(x_{1}\right), \quad \forall n \in \mathbb{N}
\end{array}\right.
$$

where $0<1-\beta<\alpha<\frac{1}{2 \sqrt{1+L^{2}}}, \xi \in\left(0, \frac{1}{\|B\|^{2}}\right)$ and $P_{C_{n}}$ is the projection operator from $H_{1}$ into $C_{n}$ for $n \in \mathbb{N}$. Suppose that

$$
\Omega=\left\{p \in U^{-1}\left(\theta_{1}\right): A p \in \mathcal{F}(S)\right\} \neq \emptyset .
$$


(a) $x_{n} \rightarrow$ as $n \rightarrow \infty$,

(b) $A x_{n} \rightarrow$ Aq as $n \rightarrow \infty$.

Remark 4.1 In Theorems 3.1 and 4.1, the control coefficients $\alpha$ and $\beta$ can be respectively replaced with the sequences $\left\{\alpha_{n}\right\}$ and $\left\{\beta_{n}\right\}$ satisfying $0<\varepsilon<1-\beta_{n}<\alpha_{n}<\frac{1}{2 \sqrt{1+L^{2}}}$ for some positive real number $\varepsilon$.

Remark 4.2 Obviously, all results in this paper are true if $H_{1}=H_{2}$. They generalize and improve many results in the literature; see, for instance, [23, 24, 26-29].

\section{Competing interests}

The authors declare that they have no competing interests.

\section{Authors' contributions}

Both authors contributed equally and significantly in writing this paper. Both authors read and approved the final manuscript.

\section{Author details}

${ }^{1}$ Department of Mathematics, Honghe University, Mengzi, Yunnan 661100, China. ${ }^{2}$ Department of Mathematics, National Kaohsiung Normal University, Kaohsiung, 824, Taiwan.

\section{Acknowledgments}

The first author was supported by the Candidate Foundation of Youth Academic Experts at Honghe University (2014HB0206); the second author was supported by Grant No. MOST 103-2115-M-017-001 of the Ministry of Science and Technology of the Republic of China.

Received: 12 July 2014 Accepted: 26 September 2014 Published: 22 Oct 2014

\section{References}

1. Censor, Y, Segal, A: The split common fixed point problem for directed operators. J. Convex Anal. 16, 587-600 (2009)

2. Moudafi, A: A note on the split common fixed-point problem for quasi-nonexpansive operators. Nonlinear Anal. 74, 4083-4087 (2011)

3. Moudafi, A: Split monotone variational inclusions. J. Optim. Theory Appl. 150, 275-283 (2011)

4. Yao, Y, Postolache, M, Liou, Y-C: Strong convergence of a self-adaptive method for the split feasibility problem. Fixed Point Theory Appl. 2013, 201 (2013)

5. Censor, Y, Gibali, A, Reich, S: Algorithms for the split variational inequality problem. Numer. Algorithms 59(2), 301-323 (2012)

6. Zhao, J, He, S: Strong convergence of the viscosity approximation process for the split common fixed-point problem of quasi-nonexpansive mapping. J. Appl. Math. 2012, Article ID 438023 (2012). doi:10.1155/2012/438023

7. He, Z: The split equilibrium problems and its convergence algorithms. J. Inequal. Appl. 2012, 162 (2012)

8. He, Z, Du, W-S: Nonlinear algorithms approach to split common solution problems. Fixed Point Theory Appl. 2012, $130(2012)$

9. He, Z, Du, W-S: On hybrid split problem and its nonlinear algorithms. Fixed Point Theory Appl. 2013, 47 (2013)

10. Li, C-I, Liou, Y-C, Yao, Y: A damped algorithm for the split feasibility and fixed point problems. J. Inequal. Appl. 2013, $379(2013)$

11. Moudafi, A: The split common fixed-point problem for demicontractive mappings. Inverse Probl. 26, 055007 (2010)

12. Ceng, L-C, Petrusel, A, Yao, J-C: Strong convergence of modified implicit iterative algorithms with perturbed mappings for continuous pseudocontractive mappings. Appl. Math. Comput. 209, 162-176 (2009)

13. Chen, $\mathrm{R}$, Song, $Y$, Zhou, $\mathrm{H}$ : Convergence theorems for implicit iteration process for a finite family of continuous pseudocontractive mappings. J. Math. Anal. Appl. 314, 701-709 (2006)

14. Chidume, CO, Souza, GD: Convergence of a Halpern-type iteration algorithm for a class of pseudo-contractive mappings. Nonlinear Anal. 69, 2286-2292 (2008)

15. Morales, $\mathrm{CH}$, Jung, JS: Convergence of paths for pseudocontractive mappings in Banach spaces. Proc. Am. Math. Soc 128, 3411-3419 (2000)

16. Yao, Y, Liou, Y-C, Chen, R: Strong convergence of an iterative algorithm for pseudocontractive mappings in Banach spaces. Nonlinear Anal. 67, 3311-3317 (2007)

17. Zhou, $\mathrm{H}$ : Strong convergence of an explicit iterative algorithm for continuous pseudo-contractions in Banach spaces Nonlinear Anal. 70, 4039-4046 (2009)

18. Schu, J: Approximating fixed points of Lipschitzian pseudocontractive mappings. Houst. J. Math. 19, 107-115 (1993)

19. Udomene, A: Path convergence, approximation of fixed points and variational solutions of Lipschitz pseudocontractions in Banach spaces. Nonlinear Anal. 67, 2403-2414 (2007)

20. Song, Y: Strong convergence of viscosity approximation methods with strong pseudocontraction for Lipschitz pseudocontractive mappings. Positivity 13, 643-655 (2009)

21. Song, Y: A note on the paper 'A new iteration process for generalized Lipschitz pseudocontractive and generalized Lipschitz accretive mappings'. Nonlinear Anal. 68, 3047-3049 (2008) 
22. Ishikawa, S: Fixed point by a new iteration method. Proc. Am. Math. Soc. 4(1), 147-150 (1974)

23. He, Z: A new iterative scheme for equilibrium problems and fixed point problems of strict pseudo-contractive mappings and its application. Math. Commun. 17, 411-422 (2012)

24. Yao, Y, Postolache, M, Liou, Y-C: Coupling Ishikawa algorithms with hybrid techniques for pseudocontractive mappings. Fixed Point Theory Appl. 2013, 211 (2013)

25. Du, W-S, He, Z: Feasible iterative algorithms for split common solution problems. J. Nonlinear Convex Anal. (in press)

26. Zhou, H: Convergence theorems of fixed points for Lipschitz pseudo-contractions in Hilbert spaces. J. Math. Anal. Appl. 343, 546-556 (2008)

27. Tang, YC, Peng, JG, Liu, LW: Strong convergence theorem for pseudocontractive mappings in Hilbert spaces. Nonlinear Anal. 74, 380-385 (2011)

28. Chidume, $\mathrm{CE}$, Zegeye, $\mathrm{H}$ : Approximate fixed point sequences and convergence theorems for Lipschitz pseudo-contractive maps. Proc. Am. Math. Soc. 132, 831-840 (2003)

29. Shahzad, N, Zegeye, $\mathrm{H}$ : Approximating a common point of fixed points of a pseudocontractive mapping and zeros of sum of monotone mappings. Fixed Point Theory Appl. 2014, 85 (2014)

10.1186/1687-1812-2014-219

Cite this article as: He and Du: On split common solution problems: new nonlinear feasible algorithms, strong convergence results and their applications. Fixed Point Theory and Applications 2014, 2014:219

\section{Submit your manuscript to a SpringerOpen ${ }^{\circ}$ journal and benefit from:}

- Convenient online submission

- Rigorous peer review

- Immediate publication on acceptance

- Open access: articles freely available online

- High visibility within the field

- Retaining the copyright to your article 